\title{
Short-term collisional evolution of a disc perturbed by a giant-planet embryo
}

\author{
S. Charnoz ${ }^{1}$, P. Thébault ${ }^{2}$, and A. Brahic ${ }^{1}$ \\ 1 Université Paris 7 Denis Diderot, Service d'Astrophysique \& CEA Saclay, France \\ 2 DESPA, Observatoire de Paris, 92195 Meudon Cedex Principal, France
}

Received 26 December 2000 / Accepted 9 April 2001

\begin{abstract}
A simple numerical model has been developed to study the evolution of a disc of planetesimals under mutual inelastic collisions in the potential field of a central body and of an embedded giant-planet embryo. Masses for the latter range from 0.5 to 300 Earth masses. A mass of $15 M_{\oplus}$ is typical of the solid-core model for the formation of giant planets. The initially cold disc consists of a few thousand particles. Those initially present between one and three Hill radii from the perturber's orbit are transferred to very eccentric orbits causing violent collisions throughout the disc. The perturbation propagates far from the perturber, like a heat transfer: a $15 M_{\oplus}$ perturber orbiting at 5.2 a.u. heats up the disc from 2.3 to at least 11 a.u. from the central body in a few $10^{5}$ to $10^{6}$ years. Relative velocities are typically increased by a factor of 10 to 100 . The extent of the heated region increases with the protoplanet's mass while the propagation timescale decreases. The resulting radial mixing has potential applications for the origin of the Asteroid Belt, in particular for the radial distribution of the asteroid's spectroscopic families.
\end{abstract}

Key words. Solar System: formation - planets ans satellites: general - minor planets, asteroid

\section{Introduction}

Terrestrial planets may have formed from a swarm of accreting kilometer-sized planetesimals. Numerical simulations and analytical work have shown that accretion processes may be relatively fast, a few $10^{5}$ years, due to the so called runaway growth of a few isolated embryos (Greenberg et al. 1978; Wetherill \& Stewart 1989; Barge \& Pellat 1990; Kokubo \& Ida 2000). This fast accreting phase is characterized by the fact that a large fraction of the mass remains in the smallest kilometer-sized bodies and that the system remains "cold", with encounter velocities comparable to the escape velocities of the smaller objects.

The early stage of planetary accretion is usually studied with numerical "particle-in-a-box" simulations of the local evolution of a planetesimal swarm, using a statistical description of the dynamics of the system that can be applied to a weakly perturbed disc. Yet, growing giantplanet embryos perturb the disc at short and long ranges thus rendering this assumption invalid. Instead, the later stage of planetesimal accretion phase has been studied over the last decade with direct deterministic simulations. These studies have focused mainly on the local gravita-

Send offprint requests to: S. Charnoz,

e-mail: charnoz@discovery.saclay.cea.fr tional deflections around terrestrial-planet embryos (Ida \& Makino 1993; Tanaka \& Ida 1996) and on the long-range perturbations of proto-giant planets in the inner Solar System (Marzari \& Scholl 1997; Kortenkamp \& Wetherill 2000; Petit et al. 1999; Petit et al. 2000). These simulations have taken into account gravitational deflections and sometimes gas drag effects. Petit et al. (2000) have studied the gravitational evolution of test particles within the inner Solar System, under the influence of both lunar to martian sized embryos and a fully formed Jupiter. They have shown that the initial Asteroid Belt region becomes highly unstable, on a timescale of a few $10^{5}$ years, due to the coupled effect of the embryos' gravitational stirring and Jupiter's mean-motion resonances. This mechanism might result in a strong depletion of the Asteroid Belt and in an increase of particle eccentricities and inclinations, as observed today.

The effect of inelastic collisions (sometimes designated as "physical collisions") among planetesimals during the mid-stage of planetesimal accretion has hardly been explored, partly because of significant numerical difficulties. This effect, however, cannot be neglected because the expected collision time scale of order $10^{4}$ years (Weidenschilling 1977) is much lower than the typical timescale of runaway growth, gas drag decay and ejection time induced by neighbouring giant planets 
(Petit et al. 2000). Stern \& Weissmann (2001) stress that collisional timescales might be short enough to prevent a significant fraction of bodies deflected by Jupiter from reaching the Oort's cloud. It should be also stressed that high-velocity encounters mostly result in collisions, i.e. fragmentation or inelastic rebound, rather than in gravitational deflections or accretion, so they cannot be neglected in a highly perturbed region. This is particularly true in the vicinity of a growing planetary embryo and also close to its mean-motion resonances, where encounter velocities may far exceed the planetesimal escape velocity.

This problem has been studied, using numerical simulations, by Thébault \& Brahic (1999), who investigated the coupling of inelastic collisions and gravitational perturbations on massless test particles around the 2:1 meanmotion resonance (at 3.27 a.u.) of a $300 M_{\oplus}$ proto-Jupiter. Their main result is that high velocity collisions stir the disc and thus propagate the resonant perturbation inwards over $\sim 1.2$ a.u. in $10^{5}$ years. In the resulting perturbed region, encounter velocities reach a few $100 \mathrm{~m} / \mathrm{s}$. Marzari \& Scholl (2000) have chosen the same deterministic approach to study the effect of collisions and gas drag in close binary systems but in two dimensions. They have shown that collisions coupled with gaseous friction efficiently cool the disc against gravitational heating. Richardson et al. (2000) have developed a tree code simulation of a disc containing $10^{6}$ particles. Jupiter's gravitational perturbations, inter-particle gravity and perfectly accreting collisions were included. However, the latter assumption may not be valid for the most perturbed parts of the disc. Up to now, this approach is limited to the first $10^{3}$ years of evolution, which is too short with respect to both dynamical and accretion timescales.

The present study follows the step-by-step approach of Thébault \& Brahic (1999) and of Marzari \& Scholl (2000). It uses an improved numerical model to follow 3000 to 4000 finite-radius particles suffering inelastic collisions and perturbations from a protoplanet at 5.2 a.u., with a mass ranging from 0.5 to $300 M_{\oplus}$, with a particular attention to the $10-15 \quad M_{\oplus}$ case, typical of the embryo of a giant planet. The disc extends from 2 a.u. to 11 a.u., encompassing the protoplanet itself. The initial disc is cold, i.e. random velocities are small compared to orbital velocities, and particle random velocities of $10 \mathrm{~m} / \mathrm{s}$ are comparable to the escape velocity of planetesimals in the actual protoplanetary disc. Since no actual statistical and direct simulations are able to study a perturbed and extended planetesimal disc, we deliberately study the simplest, but non-trivial, mechanical system which shares common physics with the "real" perturbed planetesimal disc. We include the embryo's gravitational perturbations with dissipative collisions among particles. Extrapolation from the model to the real planetesimal disc is performed via a scaling law on the number and on the size of particles. Indeed, it has been shown that a collisional system with a large number of small particles may be simulated with a small number of large particles (Brahic 1976). This scaling law allows us to simulate with only 3000 to
4000 particles the collisional evolution of a disc that may contain several billions of bodies. This approach has been used efficiently in the past in different astrophysical contexts: for the study of dissipative Keplerian discs (Brahic 1976), planetary rings (Hanninen \& Salo 1992, 1994; Hertzsch et al. 1997), planetesimal disc (Thébault \& Brahic 1999; Marzari \& Scholl 1997, 2000). Limitations of this approach are detailed in Sect. 2.

The questions we address are:

- What is the effect of collisions among planetesimals coupled with strong gravitational perturbations?

- What are the typical collisional timescales?

- What are the particle random velocities in the perturbed region?

- What is the spatial extent of the perturbed region?

- Is it possible to have a radial mixing of material ?

- What is the dependence on the embryo's mass?

The model and numerical algorithm are presented in Sect. 2. In Sect. 3, the collisionless evolution of the disc under the influence of the perturber is presented so as to illustrate the role of gravitational perturbations. In Sect. 4, the collisional evolution of the perturbed disc is described. Results are discussed in Sect. 5, and in particular the possible role of all non-considered mechanisms. In Sect. 6, possible implications for the Solar System formation are proposed.

\section{The model and the numerical algorithm}

\subsection{The model}

\subsubsection{Constraints on direct simulations and scaling factor}

The model is a three-dimensional disc composed of 3000 to 4000 massless particles with finite radii orbiting in the gravitational field of a central body and of a massive secondary body, which is called the "perturber" hereafter.

The advantage of such deterministic simulations lies in the possibility to take precisely into account detailed gravitational perturbations induced by the massive embryo. The disadvantage is a limited number of bodies, typically a few thousand instead of few billions that actually populated the early inner Solar System. Nevertheless, the global collisional evolution of a highly populated system may be simulated with a small number of large particles if the collision rate is the same in the "real" system and in the simulation (Brahic 1976; Trulsen 1971). This constraint is satisfied as long as the total geometrical cross sections $N R^{2}$ (where $N$ is the total number of bodies and $R$ their radius) of both the numerical and the "real" system are equal (Brahic 1976; Hanninen \& Salo 1992, 1994; Marzari \& Scholl 2000). Note that $N R^{2}$ equals the opticaldepth times a constant multiplicative factor, which only depends on the system's size. As a consequence, collision rates being equal in both systems, their evolution is similar as long as other interactions, like mutual gravitational 

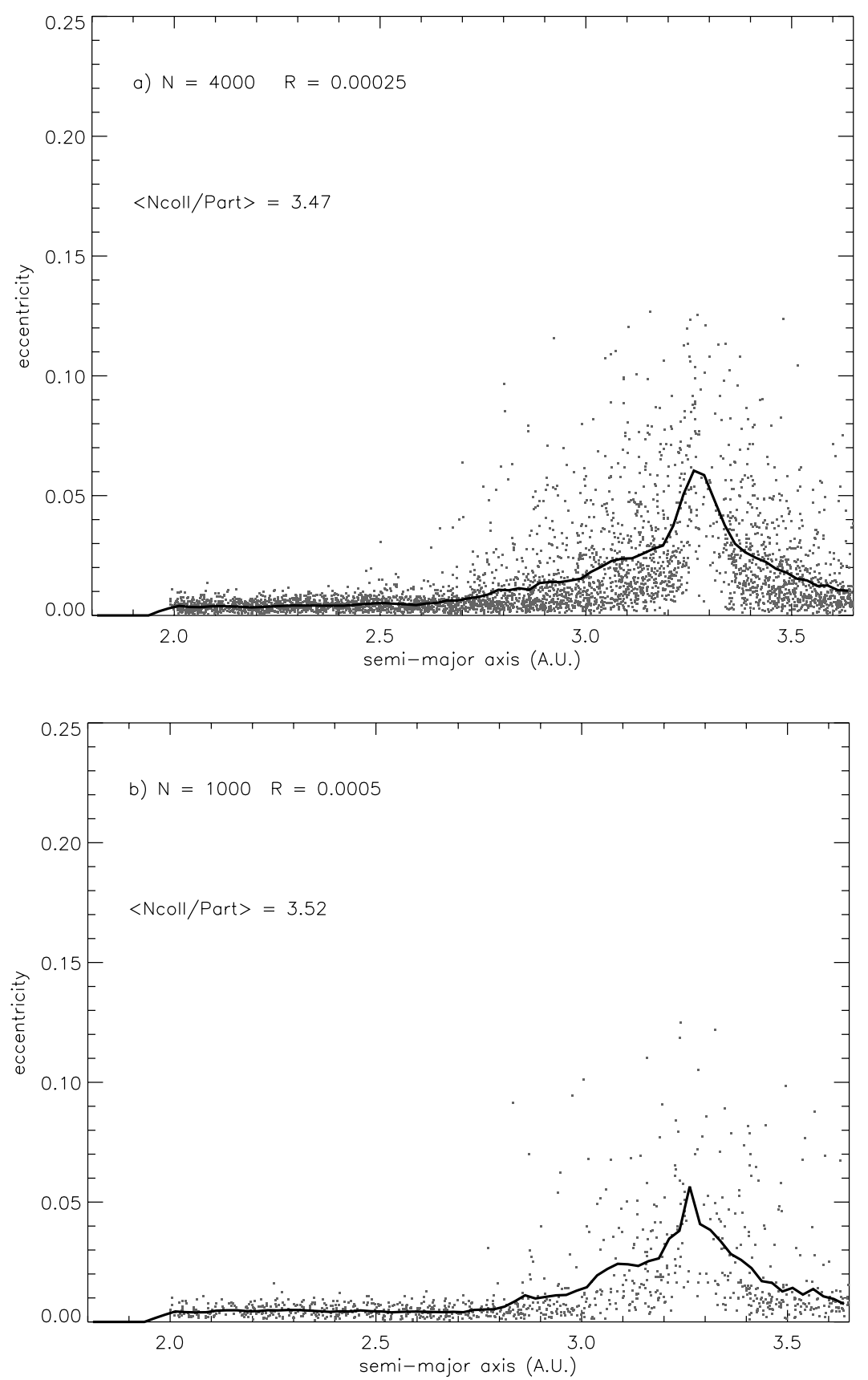

Fig. 1. Collisional evolution of finite-radius particles after $50 \times 10^{3}$ years of evolution, close to the $2: 1$ mean-motion resonance of Jupiter (3.27 a.u.), in two different simulations with different particle radii $(R)$ and different number of particles $(N)$, but the same optical depth. The comparison between the two figures shows a similar evolution since the average number of collisions per particle remains nearly the same (Ncoll/Part).The black line shows the local median eccentricity.

deflections, can be neglected. Two further conditions must be met to avoid numerical artefacts. First, to avoid finite size effects, the particle radius must be much smaller than any characteristic lengthscale of the system such as the size of the system, the width of the perturber Hill's radius or of its mean-motion resonances. Second, the particle surface density should be large enough to avoid strong statistical noise. To illustrate the validity of these arguments Fig. 1 presents test simulations that clearly illustrate the identical behaviour of two dynamically perturbed systems with same optical depth but different $N$ and $R$.

\subsubsection{Characteristics of the model}

Several mechanisms that may be relevant for the early Solar System are deliberately neglected in the framework of our step-by-step study, which focuses currently on dissipative collisions coupled with proto-Jupiter's gravitational perturbations. As a consequence of the previous Sect. (2.1.1) the following assumptions are used in the present study:

- No gravitational deflections among particles: This assumption is realistic in a disc perturbed by a 
proto-Jupiter, as shown a posteriori in Sect. 5.2. In addition, this is a condition of validity of the scalinglaw described in Sect. 2.1.1;

- The particle's radius is $5 \times 10^{-4}$ a.u.: This value is chosen so that the collision rate among particles is about 1 collision per particle every $10^{4}$ years at 3 a.u. away from the central body. This is in agreement with the collision timescale that may be expected initially in the region of the actual Asteroid Belt, assuming that about $5 M_{\oplus}$ of kilometer-sized planetesimals were initially present in this region (assuming $\rho=3 \mathrm{~g} / \mathrm{cm}^{3}$ ) with random velocities of $\sim 10 \mathrm{~m} / \mathrm{s}$. One particle in the simulation does not represent one planetesimal (see Sect. 2.1.1), it is why the radius may appear unrealistic (it is comparable to the actual size of Jupiter). One particle is just the member of a dynamical system, which is believed to share a similar physical behavior with the actual planetesimal disc. The particle's radius is a scaling factor that reproduces the real collision rate and does not influence the dynamical outcomes of collisions. Because of the poor knowledge of the initial state of the actual planetesimal swarm, the collision rate is somewhat uncertain. Thus, it should rather be considered merely as a scaling factor from which the timescales of the present study are derived.

- Equal-mass bodies: Direct simulations cannot handle enough particles to consider a realistic size distribution. In addition, taking into account a size distribution would make ambiguous the definition of the optical depth, not suitable for the scaling law described in Sect. 2.1.1. Thus, the chosen radius implies that we only study the population of kilometer-sized bodies, which contains most of the mass and of the cross-section in the actual planetesimal disc. Size distribution will be included as the next-step of our stepby-step study.

- Particles suffer inelastic rebounds: Inelasticity of collisions is taken into account in the form of a radial restitution coefficient, $\epsilon_{\mathrm{r}}$ (Brahic 1976) set to 0.3. This implies a $90 \%$ kinetic energy dissipation in the radial direction, which is qualitatively in agreement with expected dissipation rate during high velocity impacts (Petit \& Farinella 1993).

- No Fragmentation: Not considering fragmentation is a somewhat unrealistic approximation when one extrapolates the results of the simulations to the early Solar System, but direct simulations are not able to handle this mechanism. However, considering a simple fragmentation model would not be necessarily more realistic than inelastic rebound. Indeed, some authors have considered simple fragmentation models in direct simulations (like Beaugé \& Aarseth 1990) with a fixed number of fragments to avoid the simulation from becoming unmanageable. However, because of the later assumption, collision rates among particles of different sizes are not respected, that is in contradiction with the basic constraints of our approach (see Sect. 2.1.1). During a fragmentation, nu- merical experiments have shown that most material is ejected in a plane roughly at righ angle to the collision axis (Nakamura \& Fujirawa 1991; Giblin et al. 1994; Martelli et al. 1994) that is dynamically very different from rebound trajectories. However, since the collision axes are isotropically distributed over many collisions, the fragments of many collisions should be also isotropically distributed. In conclusion, whereas inelastic rebound (rather than fragmentation) is unrealistic in high-velocity impacts, post-impact trajectories of both particles may be considered as tracers of fragment's trajectories In Sect. 5.3 the possible consequences of fragmentation on planetary formation are discussed.

- No Accretion: Accretion is only possible if impact velocities are very low in a kilometer-sized planetesimal disc. As shown in Sect. 4.1.3, the proto-Jupiter will enhance encounter velocities far beyond planetesimal's escape-velocity in a very short timescale making accretion inefficient. In addition, accretion is a rare event for the population of kilometer-sized planetesimals. Thus it may not have any influence within the timescales we consider here.

Initial conditions are chosen to reproduce the initial dynamical state of a cold disc of kilometer-sized planetesimals perturbed by a giant-planet embryo. They are listed below:

- The perturber is on a circular orbit at 5.2 a.u. $\left(a_{\mathrm{p}}\right)$. It is in agreement with current models of planetary formation that predict that runaway embryos will be on almost circular orbits because of dynamical friction (Wetherill \& Stewart 1989; Barge \& Pellat 1990; Kokubo \& Ida 2000). The mass of the perturber, $M_{\mathrm{p}}$, is constant and chosen between $0.5 M_{\oplus}$ and $300 M_{\oplus}$. A $10-15 M_{\oplus}$ perturber is considered as the Standard Case and is investigated in details since it is thought to be the typical mass of a giant-planet embryo (Pollack et al. 1996). The perturber is considered to be pointlike, thus there is no collision between the perturber and the particles.

- The particles disc has a width of 3 to 6 a.u. and lies inside or outside the perturber's orbit.

- Orbital elements of particles are chosen at random so that random velocities are about the escape velocity of planetesimals $(\sim 10 \mathrm{~m} / \mathrm{s})$, which corresponds to the initial state of an unperturbed planetesimal disc (Wetherill \& Stewart 1989). This yields small initial values of eccentricities and inclinations, of the order of $10^{-4}$ to $10^{-3}$.

- The surface density decreases by the power - 1 of heliocentric distance.

\subsection{Numerical procedure}

The motion of particles is numerically integrated by fully taking into account gravitational forces of both the central body and of the perturber. The whole system evolves using 
a fixed time-step dT. Each one is divided into two substeps: (1) integration of motion (2) detection and treatment of collisions.

\subsubsection{Integration of motion}

Particles' positions and velocities are described using the usual three-dimensional Cartesian coordinates system. Trajectories are integrated with a fixed time-step, dT, using a Bulirsch-Stoer algorithm (Press et al. 1992), which is known to be fast and reasonably accurate (Murison 1989). This integrator was preferred to a simplectic integrator like the Mixed Variable Simplectic (MVS) integrator (Wisdom \& Holman 1991) because the nominal timestep of the MVS is much too large for efficient collision detection. The nominal order of the Bulirsch-Stoer is high (13th order) so as to maintain the error on particle positions well below their radius over the typical collision timescale. We checked that the absolute error on particle positions is at worst $10^{-5}$ a.u. over 10000 years in the whole system. Jacobi's constant of particles (i.e. the energy in the reference frame rotating with the perturber) is preserved within an error of $10^{-8}$ in the absence of inelastic collision.

\subsubsection{Detection of collisions}

Whereas the time-step of a Bulirsch-Stoer is shorter than for a MVS integrator, its nominal value is about 0.1 orbital period. This is much too long for the "classic" collision detection algorithm, based on a second or third-order polynomial expansion of particle trajectories (Brahic 1976; Hanninen \& Salo 1992, 1994; Richardson et al. 2000) to work efficiently. The following improved algorithm was built and especially designed to be used with a BulirschStoer integrator.

The whole system is first integrated from $T$ to $T+\mathrm{d} T$ not considering collisions. Particle pairs that had a close encounter during the latter time-step are then detected. To perform this with little extra computation time we take advantage of the fact that a $N$ th order Bulirsch-Stoer time-step is divided into $N$ equal smaller time-steps (if based on the Stoerm's Rule or $2 N$ if based on the modified mid-point method, see Press et al. 1992). A $N$ th order polynomial is built ( $N=13$ in our simulations) for all particles to determine at any times inside the timestep, the mutual distance between neighboring particles. The intrinsic error of the $N$ th order polynomial is extracted from the Bulirsch-Stoer error checking routine. The distance of closest approach of two particles is determined by finding iteratively the minimum of function $f(t)=\left(R_{1}(t)-R_{2}(t)\right)^{2}$ where $R_{1}(t)$ and $R_{2}(t)$ are the position vectors of particles 1 and 2 at time $t$ respectively and are calculated using the $N$ th order polynomial of each particle. All particle pairs which distance of closest approach is lower than the sum of their radii plus the polynomial's error, are selected as "candidate pairs".
Exact collision times are only calculated for the candidate pairs, with the "classic" collision detection algorithm using a fourth order Runge-Kutta with a tiny time-step (lower than $10^{-3}$ of the orbital period). Colliding particles' trajectories are integrated up to the exact collision time, then collisions are processed and motions are integrated again up to $T+\mathrm{d} T$.

This method is significantly faster than the classic scheme partly because the number of candidate pairs is negligible compared to the total number of neighbouring particles. In addition, the use of a long nominal time-step of the order of 0.1 orbital period is now made possible. This saves significant computer time. In fact, the "classic" method can only work with a very small nominal time-step that has to be smaller than $10 \%$ of the one used in our new method. Tests showed that the net gain in computation time is a factor of 2 to 3 , rather than 10 because one Bulirsch-Stoer iteration needs more computational effort than an explicit integrator like a Runge-Kutta or a Leap-Frog.

\section{Disc's evolution without collision}

To get a clear understanding of the dynamical evolution of the model, we present here a description of the well-known behavior of a collisionless particle disc under the influence of a perturber. We show why collisions are expected to play an important role on the short term evolution.

\subsection{Evolution of particles' eccentricity and inclination}

Particle eccentricities and inclinations are initially in the range of $10^{-4}$ to $10^{-3}$. However, under the influence of a $15 M_{\oplus}$ perturber, eccentricities increase quickly and several dynamical structures clearly appear (Fig. 2). They may be classified into three qualitatively different regions, characterized by the Jacobi energy $\left(E_{\mathrm{j}}\right)$ of the particles (Hayashi et al. 1977; Ida \& Makino 1993), given by:

$E_{\mathrm{j}}=\frac{-1}{2 a}-a^{1 / 2}\left(1-e^{2}\right)^{1 / 2} \cos (i)+U$

where $a, e$ and $i$ are the particle's semi-major axis, eccentricity and inclination respectively. $U$ is the potential energy and is calculated in the barycentric frame centered on the Sun and rotating with the perturber. In this reference frame the central body and the perturber are located at $X=-\mu$ and $X=1$ respectively where $\mu$ equals $M_{\mathrm{p}} / M_{\mathrm{c}}$ with $M_{\mathrm{c}}$ standing for the mass of the central body. The expression of $U$ is (Hayashi et al. 1977):

$U=\frac{1}{r}-\frac{\mu}{r_{1}}-\frac{1-\mu}{r_{2}}+\frac{3}{2}+\frac{9 h^{2}}{2}-7 h^{3}+o\left(h^{3}\right)$

where $r_{1}$ and $r_{2}$ are the distance from the particle to the perturber and to the central body respectively, and $r$ is the distance from the particle to the system's barycenter; $h$ is the Hill parameter of the perturber and is equal to $a_{\mathrm{p}}(\mu / 3)^{1 / 3}$. Constant terms are chosen so that $U=0$ at Lagrange's points $L_{1}$ and $L_{2}$ of the perturber. 


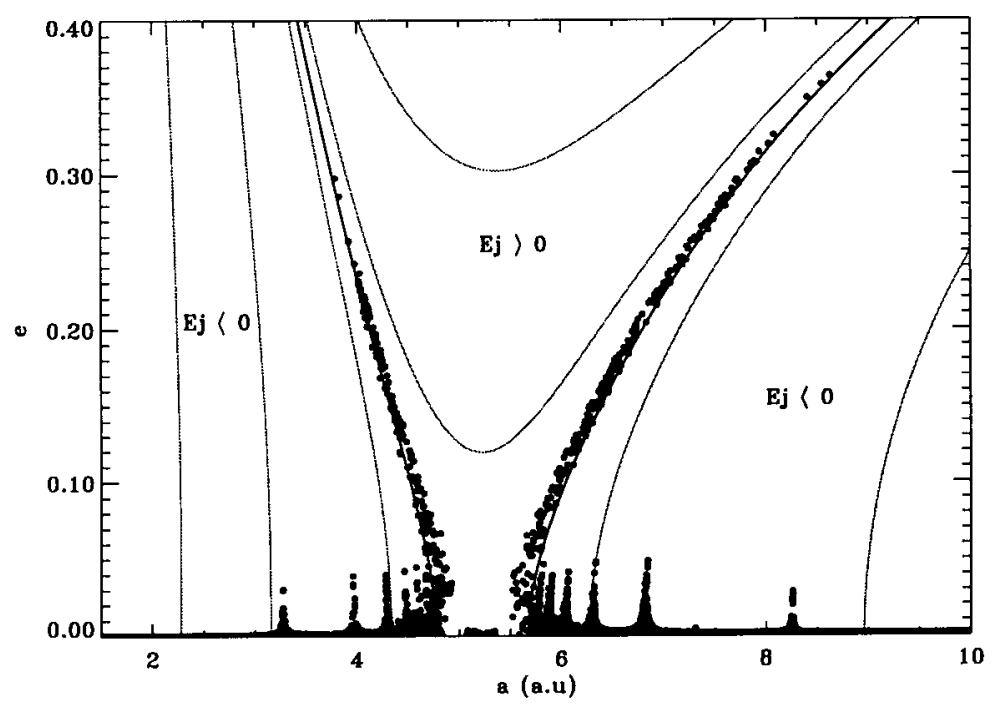

Fig. 2. Eccentricity of 4000 particles after 1000 orbital periods of a $15 M_{\oplus}$ perturber located at 5.2 a.u. on a circular orbit. Each point represents one particle. Lines are contours of constant Jacobi energy. The thick contour is the $E_{\mathrm{j}}=0$ contour.

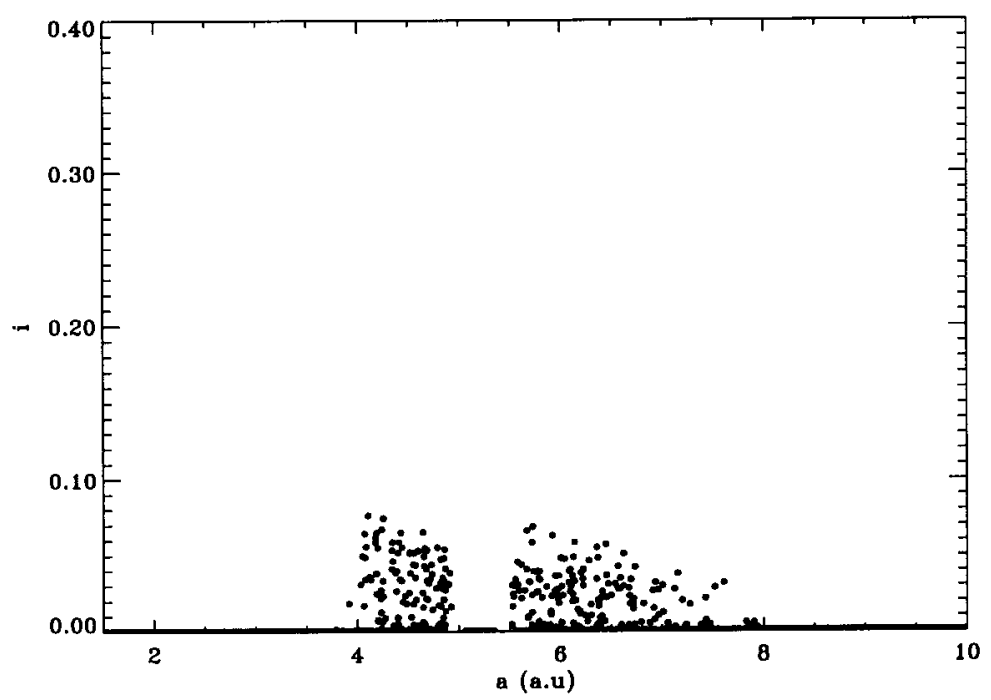

Fig. 3. Inclination of 4000 particles after 1000 orbital periods of a $15 M_{\oplus}$ perturber located at 5.2 a.u. on a circular orbit. Each point stands for one particle.

The three regions are the following:

1. The distant region $\left(E_{\mathrm{j}}<0\right)$. Far away from the perturber, particle eccentricities are weakly enhanced $\left(e \leq 10^{-3}\right)$, with the important exception of discrete narrow regions corresponding to the mean-motion resonances. Because of the low mass of the perturber, of the short period of time considered and of the circularity of perturber's orbit, only first-order mean-motion resonances appear;

2. The feeding zone region $\left(E_{\mathrm{j}}>0\right)$. Particles have the possibility to enter inside the Hill sphere of the planet (Hayashi et al. 1977), and then may be ejected from the system or accreted by the planet. They can survive in this region with a very low eccentricity inside the 1:1 corotation resonance with the perturber. This region extends typically between semi-major axes $a_{\mathrm{p}}(1-h)$ and $a_{\mathrm{p}}(1+h)$;

3. The region located along the $E_{\mathrm{j}}=0$ line is the most perturbed and has a clear "antenna" shape rooted at semi-major axes $a_{\mathrm{p}}(1 \pm 3 h)$ along the region of overlapping resonances (it is a chaotic region as shown by Wisdom 1980). Particles with a small positive $E_{\mathrm{j}}$ may closely approach the perturber and are then quickly scattered: their eccentricities may reach 0.2 or 0.3 in a few orbital periods. These features were also observed by Ida \& Makino (1993) with a full $N$-body calculation of particles interacting with a protoplanet.

The inclination diagram (Fig. 3) is in sharp contrast with the $(a, e)$ graph (Fig. 2). Indeed particle's inclinations remain mostly unchanged because of (i) the non-inclined orbit of the perturber (ii) the low initial inclinations of 


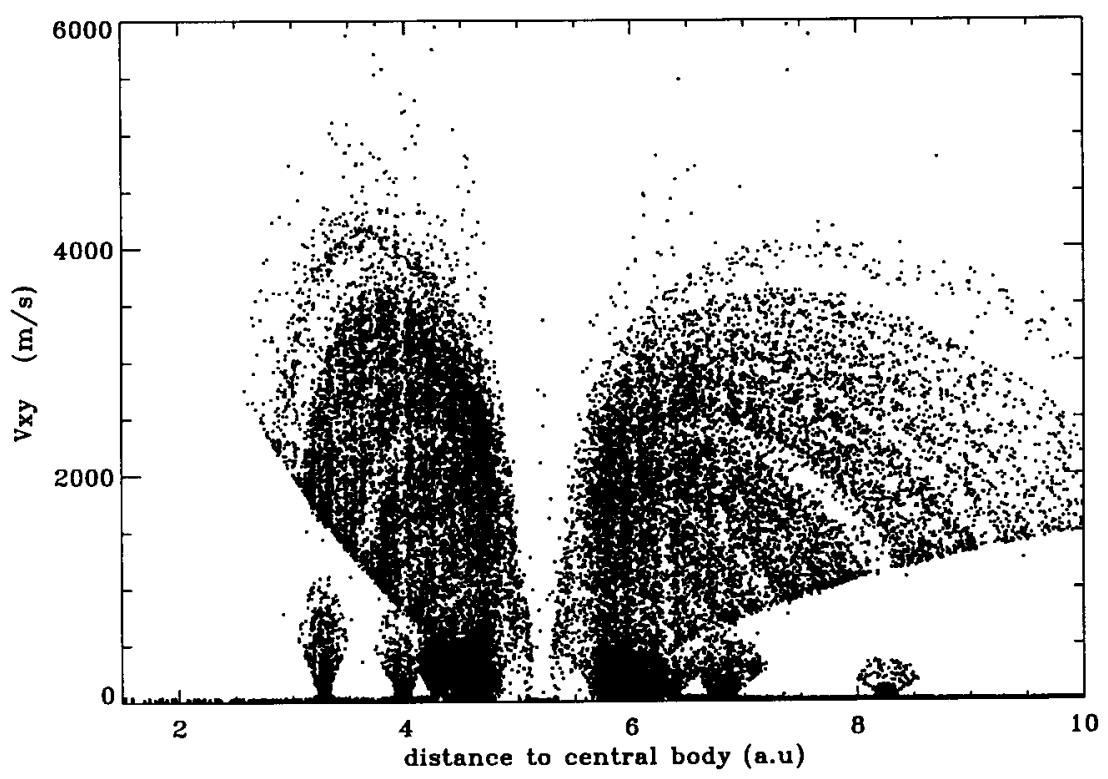

Fig. 4. Encounter velocities in the collisionless disc, projected onto the mid-plane (in-plane direction). Each point stands for a close encounter between two particles, but the collision itself is not processed. The perturber's mass is $15 M_{\oplus}$.

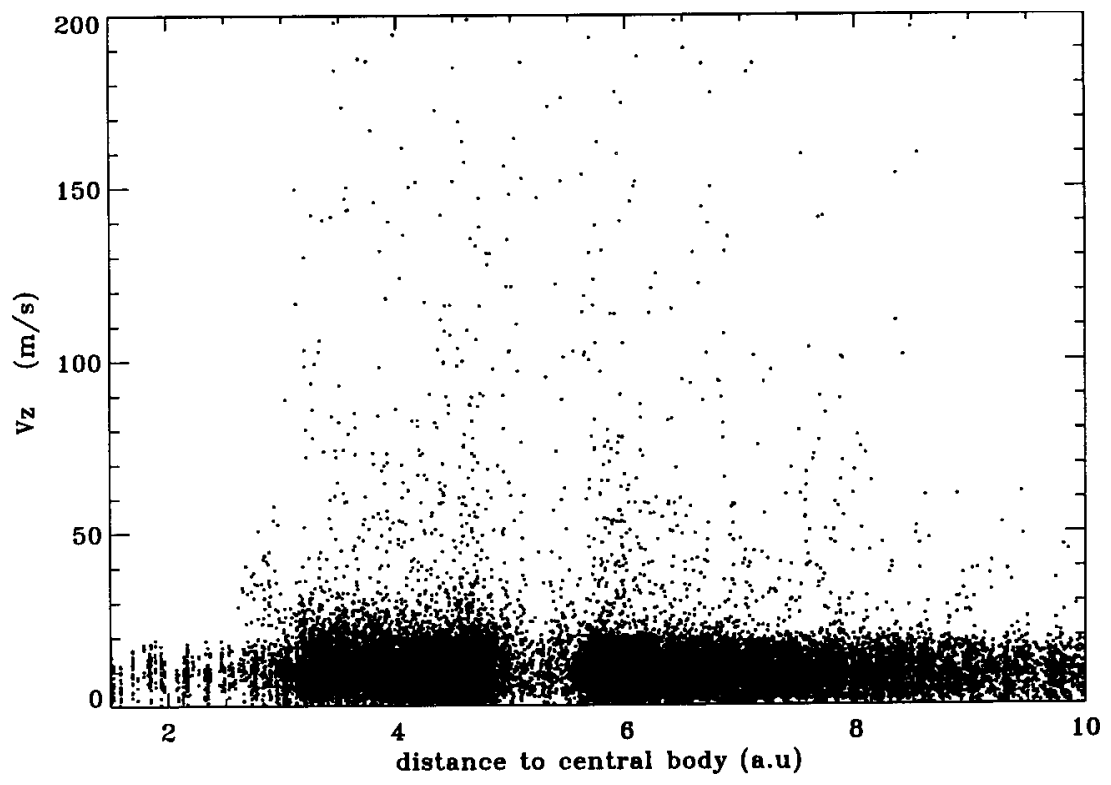

Fig. 5. Encounter velocities in the collisionless disc, projected onto the vertical axis of the disc (out-plane direction). The perturber's mass is $15 M_{\oplus}$.

planetesimals. Only some bodies, which have been strongly deflected along the $E_{\mathrm{j}}=0$ line, have their inclination significantly increased. The usual approximation $i \sim e / 2$, valid in a collisionally relaxed disc (Barge \& Pellat 1990) is not valid any more in the perturbed disc, and the collision rate is no longer proportional to the optical depth (see below).

\subsection{Relative velocities and encounter rate}

The inplane and outplane magnitude of encounter velocities in the collisionless disc are displayed in Figs. 4 and 5 respectively. A "butterfly wings" structure is present in
Fig. 4, extending on both sides of the perturber's location. These are the high velocity encounters, of the order of $2000 \mathrm{~m} / \mathrm{s}$, which involve at least one particle strongly deflected by the perturber along the $E_{\mathrm{j}}=0$ line. Because of large eccentricities, high velocity encounters take place in a wide region extending from 2.5 a.u. to 10 a.u. Numerous low velocity encounters, involving weakly perturbed bodies are also present. The resulting encounter speed is of the order of $10 \mathrm{~m} / \mathrm{s}$, which is comparable to its initial value.

Encounters velocities in the out-plane direction (Fig. 5) are 100 to 1000 times weaker than in the in-plane direction. This anisotropy reflects the asymmetry between particles' eccentricity and inclination. Consequently the 
perturbed disc is like a "thin hot layer". This behaviour is the consequence of the initial state of the planetesimal disc, in which inclinations are initially very low $\left(\sim 10^{-4}\right)$. Perturber's gravitational perturbations induce a two-dimensional dynamics on a short-term evolution, which consequences are detailed below.

The "particle-in-a-box" encounter rate per time unit, per cross section unit, and normalized to one target body and one projectile is (Spaute et al. 1991):

$$
N_{\mathrm{c}}=\frac{V_{\mathrm{e}}}{V}
$$

where $V$ is the volume of space occupied by the population of particles, and $V_{\mathrm{e}}$ the impact velocity. It is approximated by $e V_{\text {circ }}$, where $V_{\text {circ }}$ is the circular orbital velocity . For an extended population in semi-major axes $(\Delta a>2 a e)$, $V$ is approximated by:

$$
V \sim 4 \pi a^{2}(\Delta a) i
$$

$N_{\mathrm{c}}$ is then proportional to $e / i$. In a collisionally relaxed disc, $e / i$ is a constant factor of the order of 1 and the encounter rate only depends on the optical depth and on the orbital period. However, the encounter rate is strongly enhanced here by the asymmetry between $e$ and $i$. In Figs. 4 and 5 the encounter rate is about one encounter per particle every $\sim 200$ years between 2 and 4 a.u., rather than one encounter per particle every $10^{4}$ years in an unperturbed disc. In conclusion, one may expect inelastic collisions to play an important role on the short-term evolution: this is the subject of the following section.

\section{Collisional evolution of the disc}

\subsection{Standard case: $A 10$ to $15 M_{\oplus}$ perturber}

\subsubsection{Evolution of eccentricities and inclinations in the inner disc}

The collisional evolution of the inner disc shows significant departure from the collisionless case very early (Fig. 6). In this simulation, the perturber's mass is $15 M_{\oplus}$. After only 2000 years, the $E_{\mathrm{j}}=0$ wings as well as the $2: 1$ (3.27 a.u.) and 3:2 (3.9 a.u.) resonances begin to spread out. These structures are unstable against collisions, which occur at very high velocities (Fig. 4) resulting in major modifications of colliding particle's orbits. Because of their enhanced eccentricity, colliding bodies may reach initially weakly perturbed regions, where their random kinetic energy is transferred into the cold disc via multiple subsequent collisions. A collisional diffusion wave is then triggered. This is very similar to a heat transfer. This kind of mechanism was first described by Thébault \& Brahic (1999) with a $300 M_{\oplus}$ perturber in the restricted case of the 2:1 resonance as the only source of eccentric particles. Still, it is clear in Fig. 6 that the 2:1 resonance generated diffusion is a relatively marginal phenomenon compared to the main wave triggered close to the perturber along the $E_{\mathrm{j}}=0$ line.
After $1.6 \times 10^{5}$ years the collisional diffusion has reached its maximum extention down to 2.2 a.u. In this perturbed region, particle eccentricities increase linearly from 0.005 at 2.2 a.u. to 0.05 at 3.5 a.u. and are almost stable beyond. In addition, the region of overlapping resonances is almost depleted and only a few particles remain along the $E_{\mathrm{j}}=0$ line. Indeed, these particles are the most eccentric of the system and thus are in some sense the "energy tank" of the collisional diffusion. On the one hand, only one collision is necessary to remove them from the chaotic region. On the other hand, many collisions are necessary for particles with moderate eccentricity to migrate from the colder disc up to the chaotic region. In consequence, this region is progressively depleted. Further evolution of the system (a progressive damping of inclinations and eccentricities due to the inelasticity of collisions) is much slower and is not investigated in the present study.

Whereas initial inclinations were much smaller than forced eccentricities (see Sect. 3.1), they quickly increase as collisions redistribute energy in all degrees of freedom. Thus, the system evolves towards equipartition $(i \sim e / 2)$, which is almost reached after only 2000 years. This rapid equipartition strongly affects the collision rate, which is a somewhat counter-intuitive aspect of collisional diffusion: it is most of the time comparable to its value in a non-perturbed cold disc. To clearly illustrate this point, in Fig. 7 locations of collisions as a function of time during the first $5 \times 10^{4}$ years of evolution are reported. Since the particle surface density decreases as $1 / r$, there are more collisions in the inner part of the disc, which as a result appears darker in Fig. 7. During the first 5000 years of evolution, the collision rate is high in the vicinity of the chaotic region (between 4 and 5 a.u.) and as well as in the vicinity of the mean-motion resonances (at 3.27 and 3.9 a.u.) i.e. 1 collision per particle every 200 years, which is consistent with the study of Sect. 3.2. However, this period of high collision rate is short compared to the $2 \times 10^{5}$ years needed for the collisional diffusion to reach its maximum extention. Indeed, after a mere 5000 years the collision rate falls down to a value comparable to its value in an unperturbed disc i.e. about 1 collision per particle every $10^{4}$ years between 2 and 4 a.u. This rapid decrease in the collision rate reflects the increase in particle inclinations due to collisions (see Sect. 4.1.1). Thus, the disc can freely expand and the collision rate decreases proportionally to the ratio $e / i$. When equipartition is reached, no further significant evolution of the collision rate is observed.

\subsubsection{Evolution of eccentricities and inclinations in the outer disc}

As in the inner disc, a heat transfer is observed in the outer disc (see Fig. 8). In this simulation, the perturber's mass is $10 M_{\oplus}$ to shorten slightly the extent of collisional diffusion. After $10^{6}$ years of evolution (and 18 "real" days of computer time), the whole system, i.e. from 5 to 11 a.u., is perturbed. It is not clear whether it stops around $11 \mathrm{a} . \mathrm{u}$. 

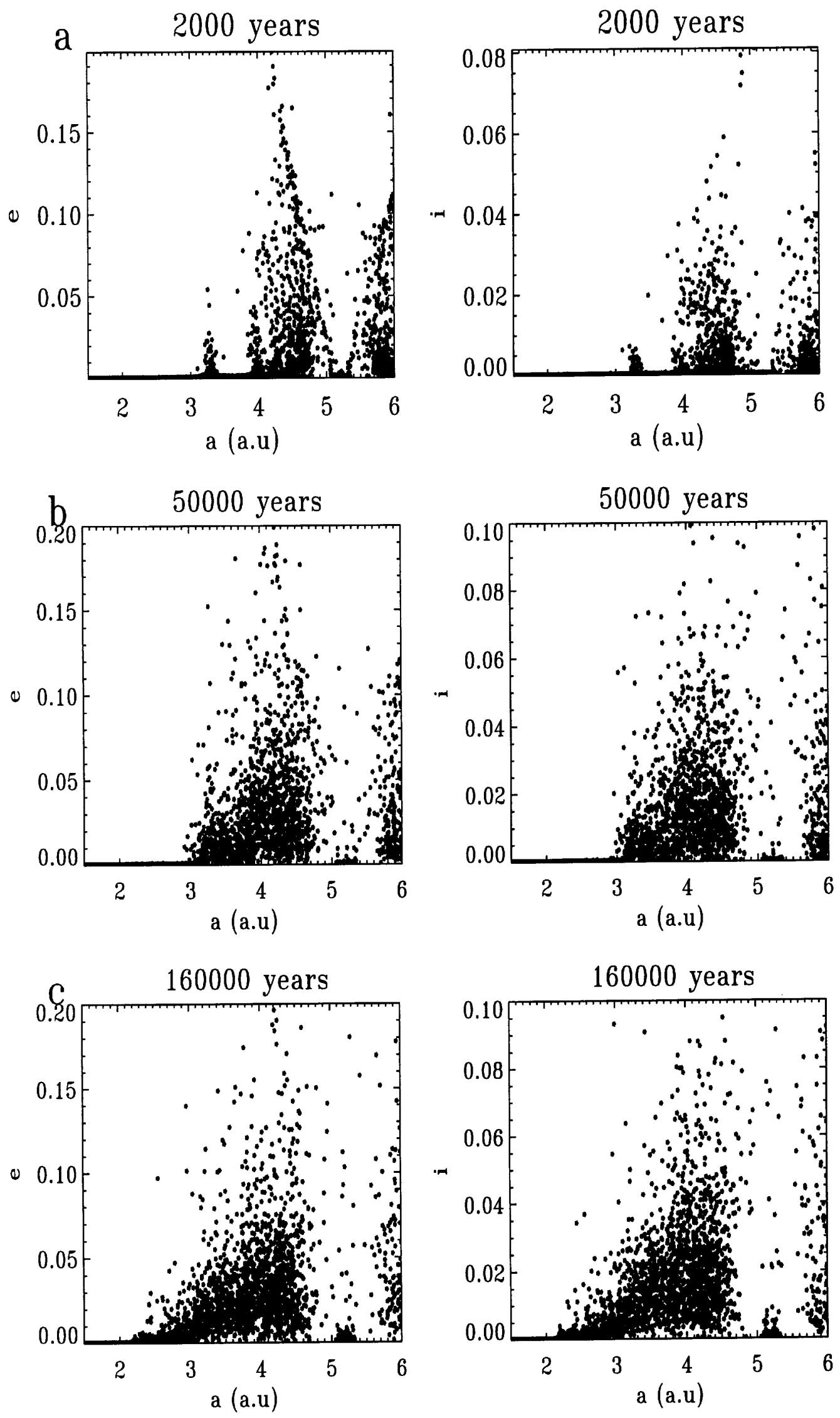

Fig. 6. Evolution of eccentricities and inclinations of particles in the inner disc at three different epochs. The $15 M_{\oplus}$ perturber is located at 5.2 a.u. on a circular orbit. 


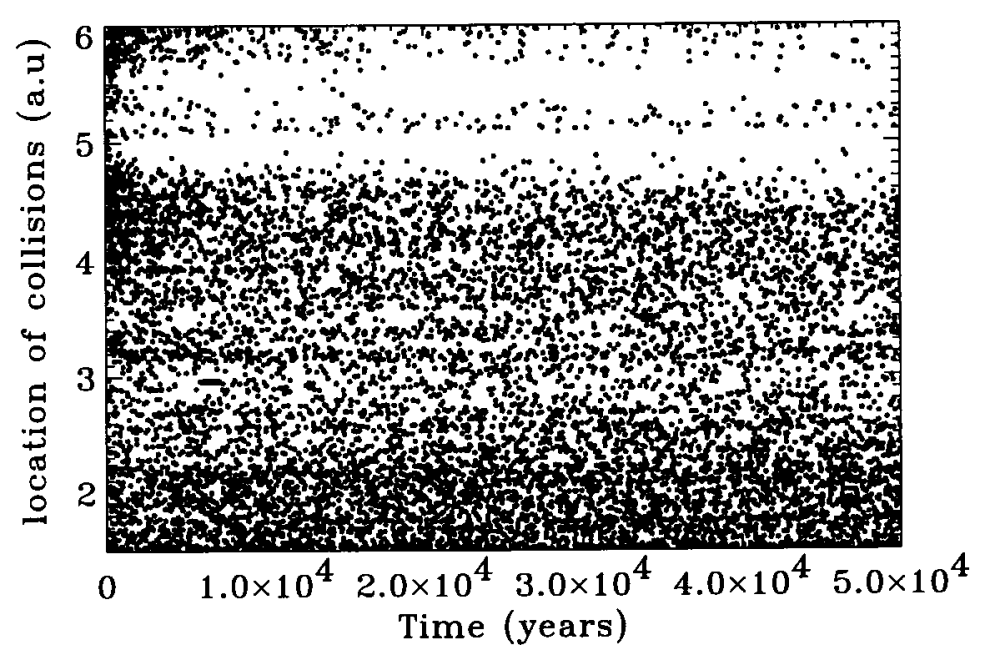

Fig. 7. Radial positions of detected collisions as a function of time. The point density is an indication of local collision rates.

or goes further beyond. However, it appears that the propagation slows down with time: the system barely changes between $6 \times 10^{5}$ and $10^{6}$ years. Thus the propagation timescale is somewhere in this range. In the perturbed region, average eccentricities range from 0.01 to 0.05 , and inclinations are about half.

An important aspect of outward collisional diffusion is that it propagates over a wide region of at least 6 a.u. whereas the inner collisional diffusion, for a same perturber's mass, extends over 2.5 a.u. only. This difference may be partly explained with simple considerations. First, as particles migrate outwards due to collisions and gravitational deflections by the perturber, their mean-free path (given by 2ae) increases linearly with their semi-major axis. This is a purely geometrical effect enhancing the extent of the outward diffusion. In addition, during a collision, an eccentric particle transfers a part of its random kinetic energy (that is of the order of $e^{2} / a$, since random velocities are of the order of $e V_{\text {circ }} \sim e / \sqrt{a}$, with $\left.G M_{\mathrm{c}}=1\right)$ to a particle initially on a circular orbit. If one considers the conservation of random kinetic energy to a first order approximation, the post-collision eccentricity of the target body is proportional to $\sqrt{a}$, which increases at larger heliocentric distances. The combination of this latter effect with the geometrical increase of mean-free path implies that a particle migrating outwards has an increasing eccentricity. This may explain the wide extent of the outward collisional diffusion.

\subsubsection{Final random velocities}

In the heated region of the inner disc, random velocities increase from $20 \mathrm{~m} / \mathrm{s}$ to $1500 \mathrm{~m} / \mathrm{s}$ at $2.2 \mathrm{a} . \mathrm{u}$. and $5 \mathrm{a} . \mathrm{u}$. respectively (Fig. 9). They are on average a few hundred meters per second, which are 10 to 100 times their initial values. Only a factor of 2 remains between out-plane and in-plane components of impact velocities. In the region of coorbital bodies, random velocities are only slightly enhanced.
The same behavior is observed in the outer disc (Fig. 10): random velocities are maximum at 6 a.u. (about $500 \mathrm{~m} / \mathrm{s}$ ) and decrease to $10 \mathrm{~m} / \mathrm{s}$ at 11 a.u. As for inward collisional diffusion, random velocities are about twice as high in the plane of the disc as in the out-plane direction. In comparison to the inner disc, there is about a factor of two difference in random velocities: this is consistent with the decrease orbital velocity at larger heliocentric distances.

\subsubsection{Radial mixing}

The collisional diffusion is also associated with a substantial radial mixing and spreading of material on a lengthscale ranging from one tenth to one whole astronomical unit (see Fig. 11). Diffusion in the inward direction is not only due to the perturber's dynamical effect, but also favored by the collision inelasticity that decreases particles' semi-major axes. Moreover, a substantial fraction of coorbital bodies has migrated inwards, down to 3 a.u. On the contrary, only a few particles below 5.2 a.u. has migrated up to the coorbital region. The region extending below 2.5 a.u. does not present any radial mixing, as the intensity of collisional diffusion is weak there.

The radial mixing in the outer disc (Fig. 12) has qualitatively the same characteristics, however with larger lengthscales because of larger heliocentric distance. Between 6 a.u. and 8 a.u., the typical mixing length is about 1.5 a.u. Particles that were initially between 5 and 6 a.u. are now spread throughout a region extending from 5 to 9 a.u. Beyond 10 a.u., the radial mixing lenghtscale is short: a mere 0.1 a.u.

\subsection{Dependence on the perturber's mass}

The size of the perturbed region as a function of the perturber's mass is quantified through multiple runs of the model with different masses ranging from $0.5 M_{\oplus}$ to $300 M_{\oplus}$. Each case is simulated at least twice with 

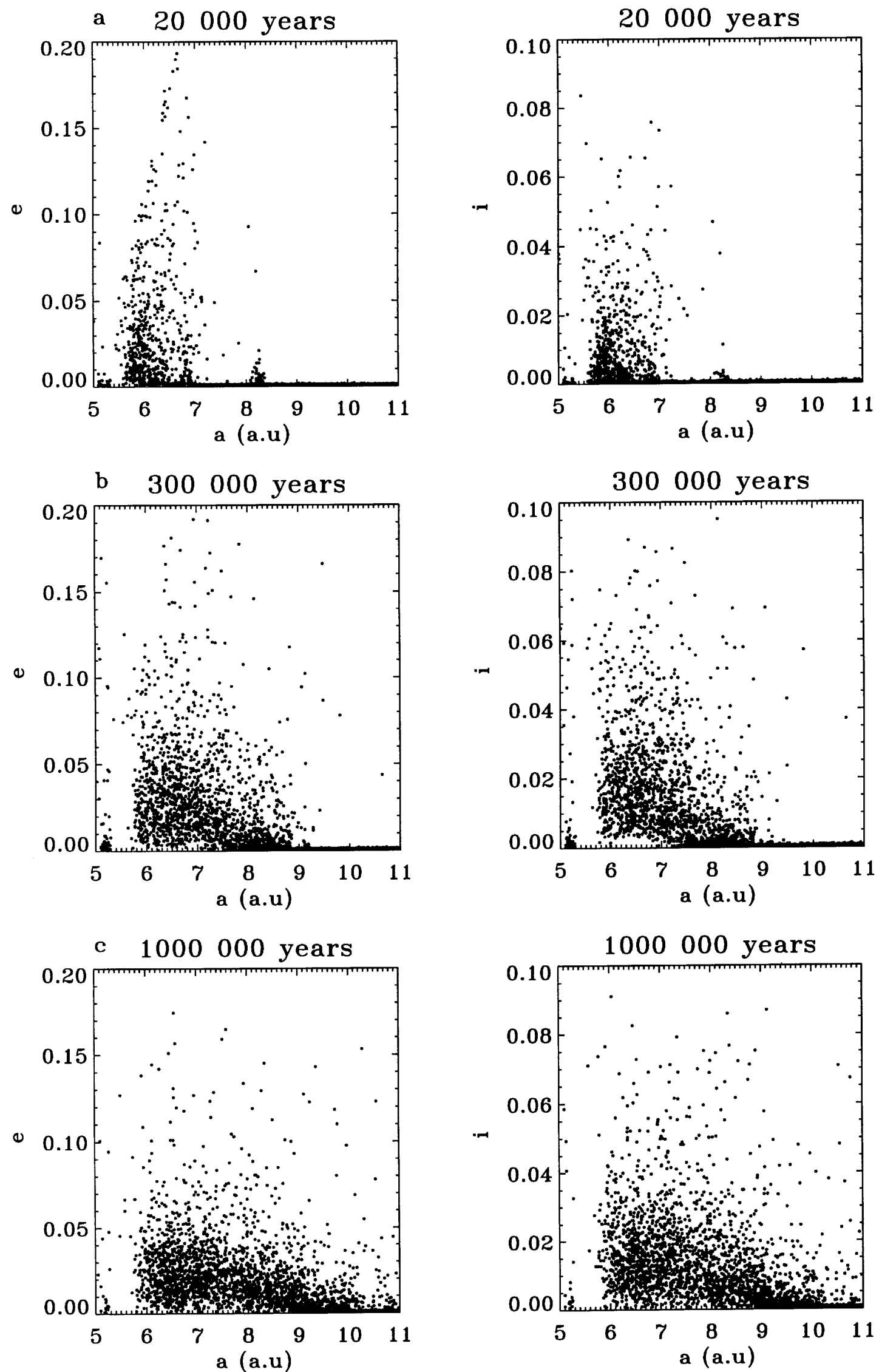

Fig. 8. Evolution of particle eccentricities and inclinations in the outer disc perturbed by a $10 M_{\oplus}$ body. 


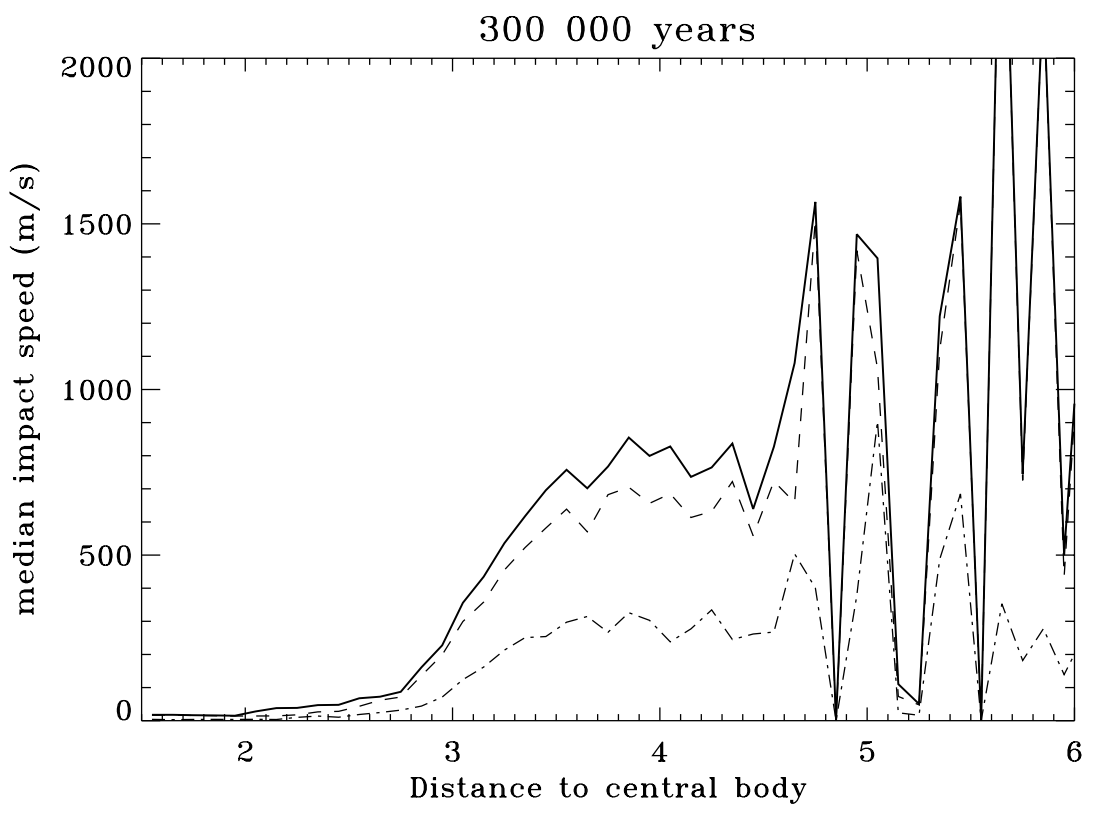

Fig. 9. Impact velocity in the disc perturbed by a $15 M_{\oplus}$ body, at the end of the collisional diffusion. The bold line shows the local median of impact velocity. The dashed line is the magnitude of impact velocity projected onto the disc's plane. The dot and dash line shows the magnitude of the impact velocity projected onto the vertical axis of the disc.

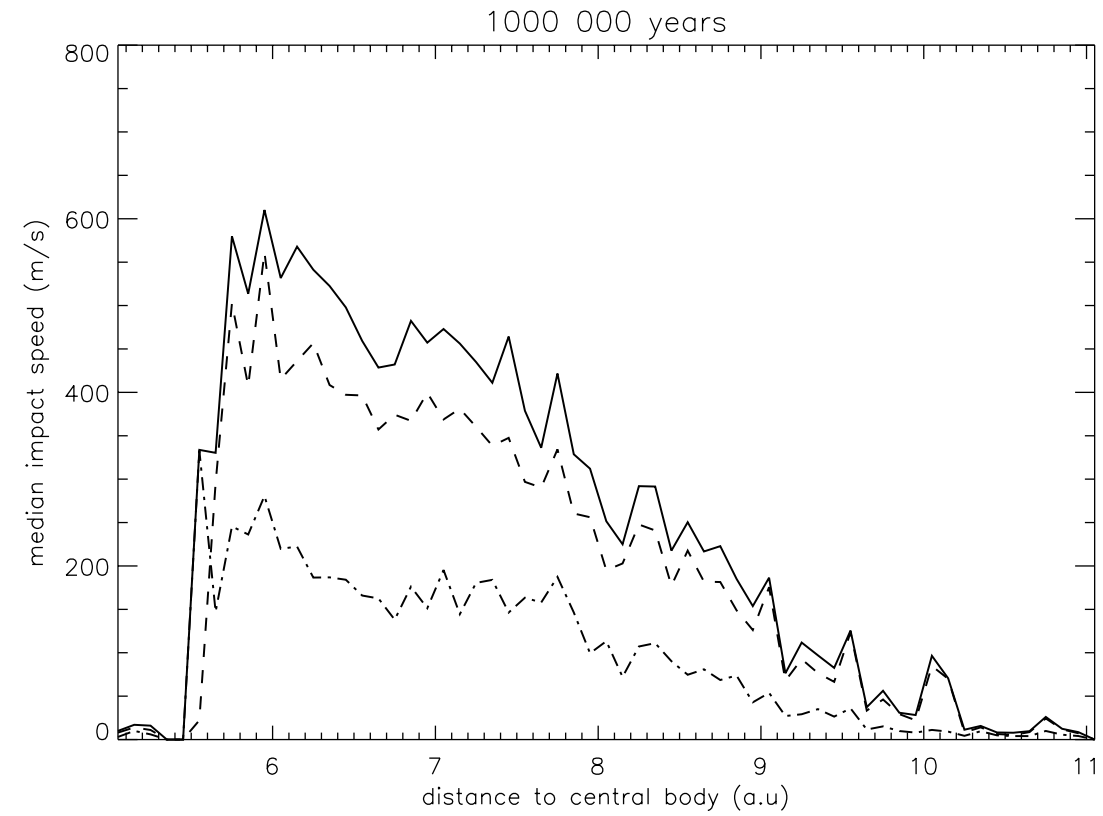

Fig. 10. Impact velocity in the outer disc perturbed by a $10 M_{\oplus}$ body at $10^{6}$ years. The bold line shows the local median of impact velocities. The dash line is the magnitude of the in-plane impact velocity. The dot and dash line is the magnitude of the out-plane impact velocity.

similar initial conditions (only initial random numbers were modified) to check that all results are stable. The inner limit of the collisional diffusion, as a function of the perturber's mass, is presented in Fig. 13. It is defined as the point where random velocities become greater than $30 \mathrm{~m} / \mathrm{s}$, chosen so that the rebound velocity is larger than the planetesimal's escape velocity. For perturber's masses ranging from 1 to $100 M_{\oplus}$, this limit shifts linearly with the logarithm of the perturber's mass. However, a kind of transition appears at $1 M_{\oplus}$ : for a perturber lighter than
$1 M_{\oplus}$, particles deflected along the $E_{\mathrm{j}}=0$ line are not eccentric enough to reach the location of the $2: 1$ and $3: 2$ mean-motion resonances. Note also that a $300 M_{\oplus}$ body perturbs the disc down to 0.9 a.u., below the Earth's orbit.

The extention of collisional diffusion in the outer disc, as a function of the perturber's mass is reported in (Table 1). Due to its large extention it was not possible to perform simulations with a massive perturber. Thus, the range of masses between $1 M_{\oplus}$ and $30 M_{\oplus}$ was studied instead. A few Earth mass body may perturb the disc up to 


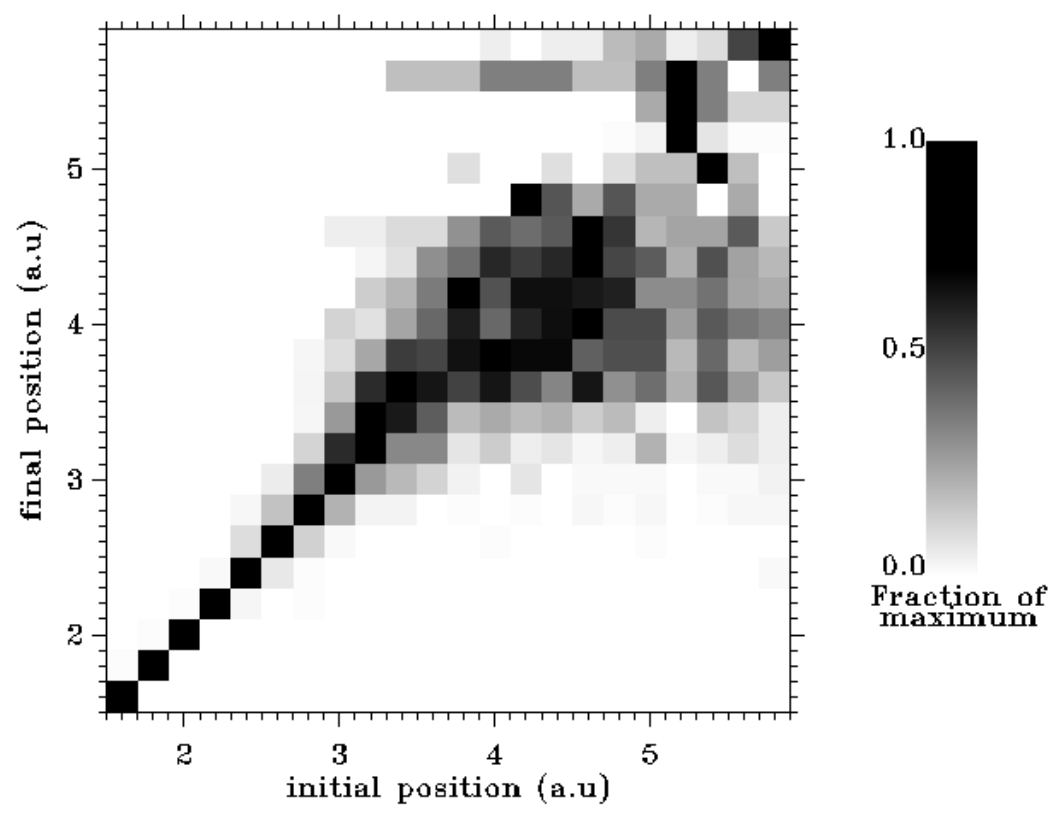

Fig. 11. Radial mixing at the end of the propagation of collisional diffusion with a $15 M_{\oplus}$ perturber. Each column is a grayscale histogram of the final semi-major axes of bodies ( $Y$ axis). Initial semi-major axes are reported on the $X$-axis. For example, bodies that were initially located at 3 a.u., are now spread between 2.5 and 4.5 a.u. at the end of the collisional diffusion. In each column, the value of the most populated bin is normalized to 1.

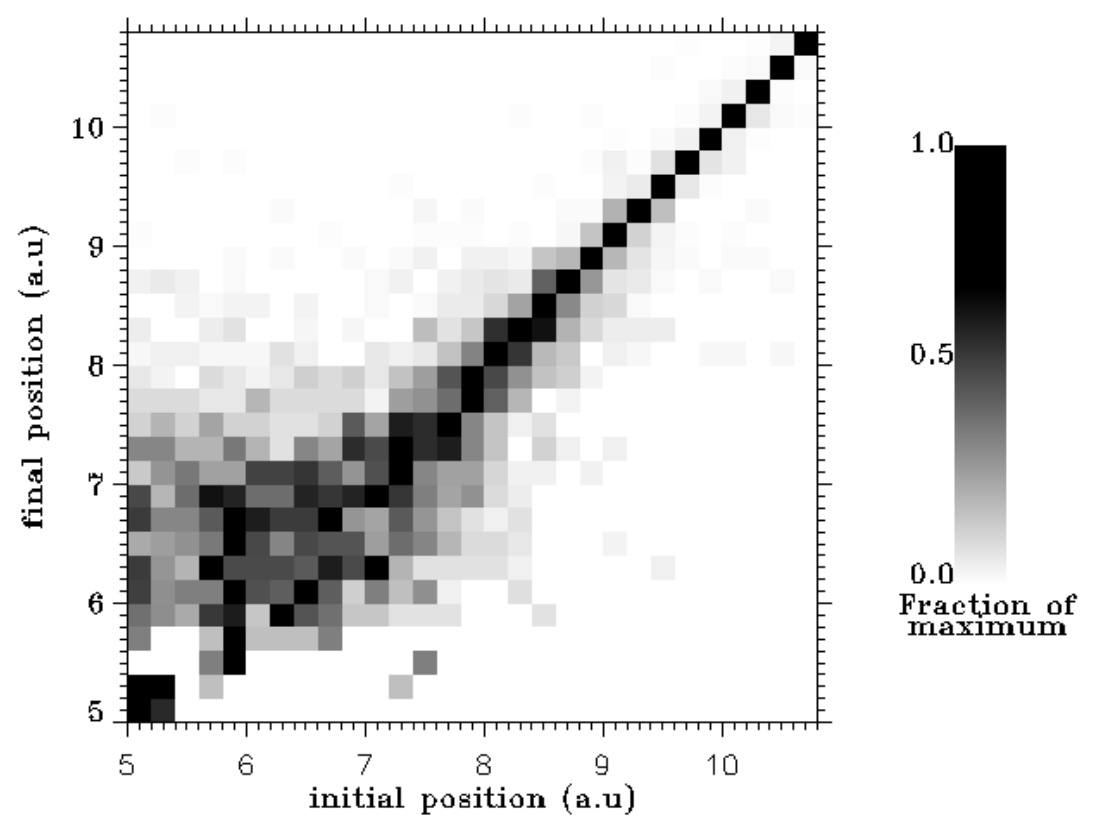

Fig. 12. Radial mixing in the outer disc, after $10^{6}$ years with a $10 M_{\oplus}$ perturber. Each column is a grayscale representation of the histogram of the final semi-major axes of bodies ( $Y$ axis) whose initial semi-major axis is on the $X$-axis.

8 a.u. from the central body, over a timescale of $10^{6}$ years. The most striking result is that a mere $1 M_{\oplus}$ perturber at 5.2 a.u. is able to heat the disc up to 7.5 a.u. over the same timescale.

\section{Possible role of additional mechanisms}

\subsection{Gas drag}

In the framework of our step-by-step study of a perturbed planetesimal disc, gas drag has been deliberately neglected since its effects have been much more investigated than collisional processes (Marzari \& Scholl 1997; Marzari \& Scholl 2000; Tanaka \& Ida 1996; Kortenkamp \& Wetherill 2000). It is well known that gas drag decreases both eccentricity and inclination of kilometer-sized bodies, and to a lesser extent, their semi-major axes. Thus collisional diffusion may be partly damped by gas drag. A simple model of gas drag will be therefore considered in a future study. Some unpublished results of P. Thébault (private communication) suggest that collisional diffusion is indeed 


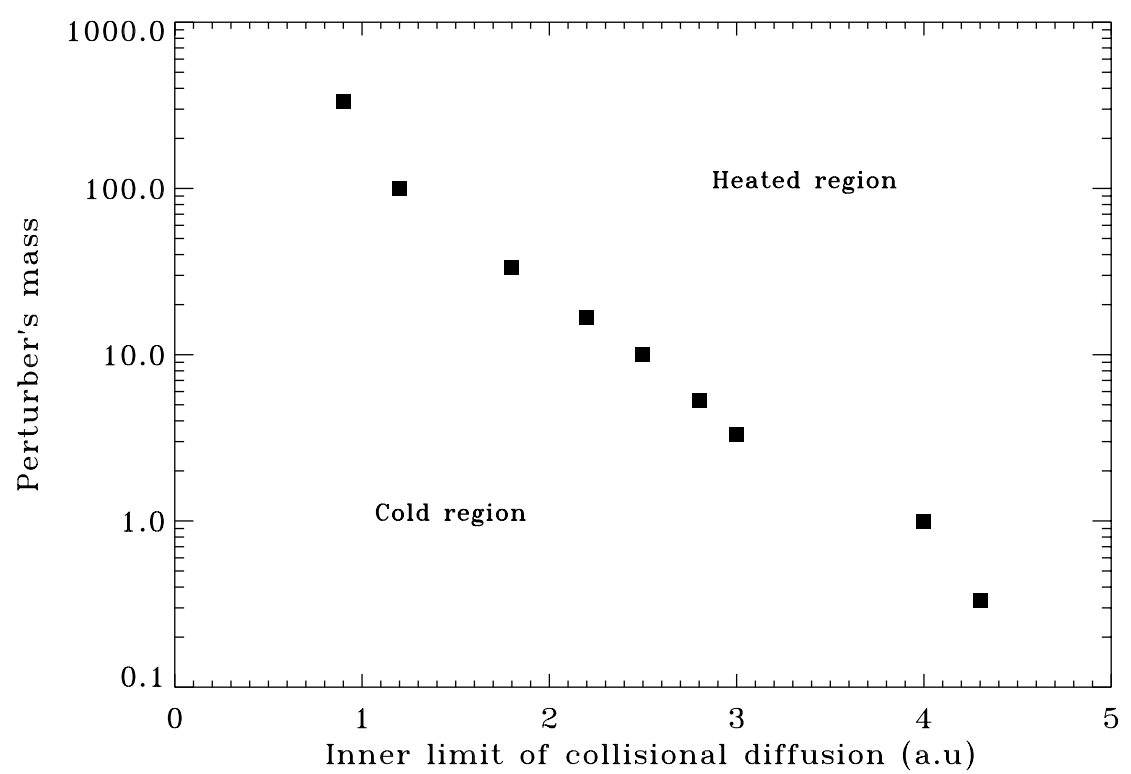

Fig. 13. Heliocentric position of the collisional diffusion inner front, as a function of the perturber's mass. The mass unit is in Earth's mass. The perturber is located at 5.2 a.u.

Table 1. Limit of outward collisional diffusion as a function of the perturber's mass. The outer limit for a 15 and $30 M_{\oplus}$ perturber may be between 12 and 20 a.u. For a $10 M_{\oplus}$ perturber it may be between 11 and 13 a.u.

\begin{tabular}{ll}
\hline $\begin{array}{l}\text { Perturber's } \\
\text { mass }\end{array}$ & $\begin{array}{l}\text { Limit of } \\
\text { collisional } \\
\text { diffusion }\end{array}$ \\
\hline $30 M_{\oplus}$ & $>>11$ a.u. \\
$15 M_{\oplus}$ & $>>11$ a.u. \\
$10 M_{\oplus}$ & $>11$ a.u. \\
$5 M_{\oplus}$ & 9 a.u. \\
$3 M_{\oplus}$ & 8.5 a.u. \\
$1 M_{\oplus}$ & 7.5 a.u. \\
\hline
\end{tabular}

substantially damped by gas drag, however its spatial extention remains comparable to the gas-free case.

\subsection{Gravitational deflections}

In the present simulations, gravitational deflections among particles were neglected, whereas in a cold disc, random energy comes mainly from gravitational deflections among planetesimals associated with differential rotation. However, as far as we are concerned with the population of kilometer-sized planetesimals, gravitational deflections may be realistically neglected since random velocities, $V_{\mathrm{r}}$, between particles are 10 to 100 times larger than kilometer-sized planetesimal escape velocity, $V_{\mathrm{e}}(\sim 10 \mathrm{~m} / \mathrm{s})$. This has two consequences:

1. The gravitational cross section becomes comparable to or smaller than the physical cross section. Indeed the two-body cross-section is approximated by (Safronov 1969):

$\sigma=\pi r^{2}\left(1+\frac{V_{\mathrm{e}}^{2}}{V^{2}}\right)$

where $r$ and $V_{\mathrm{e}}$ are the particle's radius and escape velocity respectively. The first term in the parenthesis stands for the geometrical (or "physical") cross section, and the second term stands for the gravitational cross section. If $V \geq V_{\mathrm{e}}$ then the gravitational cross section is lower than the geometrical cross section, as it is the case in the system we study;

2. During a high-speed gravitational encounter, $\Delta V_{\mathrm{r}} / V_{\mathrm{r}}$ ( $\Delta V_{\mathrm{r}}$ is the magnitude of velocity change in a given direction) is of the order of $\left(V_{\mathrm{e}} / V_{\mathrm{r}}\right)^{2}$. This is very small compared to 1. By comparison, during an inelastic collision $\Delta V_{\mathrm{r}} / V_{\mathrm{r}} \sim 1$.

For these reasons, neglecting gravitational deflections among particles is a realistic approximation in the case of a very perturbed disc, as considered here. The same approximation is done by Stern \& Weisman (2001) that study the effect of collisions involving bodies deflected by the giant planets and particles of the cold planetesimal disc.

\subsection{Fragmentation and size distribution}

Usual models of fragmentation (Petit \& Farinella 1993; Benz \& Asphaug 1999) imply that kilometer-sized basaltic bodies, colliding at velocities greater than $50 \mathrm{~m} / \mathrm{s}$, would suffer a catastrophic disruption, producing fragments with a steep size distribution. It is thus clear that our hypothesis of inelastic rebound is not realistic although the energy dissipation rate of $90 \%$ used in the simulations may be quite realistic. Bodies strongly deflected by the 
protoplanet will be catastrophically disrupted after their first collision. This bombardment takes place in the wide region in the reach of particles deflected by the perturber along the $E_{\mathrm{j}}=0$ line. For a $15 M_{\oplus}$ perturber this region extends from 3 to 10 a.u. (Fig. 2), and thus may be fed with a large population of fragments. It is then logical to ask the following question: may the fragments heat up the rest of the cold planetesimal disc? This question needs a full numerical simulation of a new type to be answered. We rather give below some simple considerations as direction for future work.

As soon as different mass bodies interact, energy equipartition takes place such that random kinetic energy balances out to $M_{1} V_{1}^{2}=M_{2} V_{2}^{2}$ where $V_{1}$ and $V_{2}$ are the random velocities of bodies with masses $M_{1}$ and $M_{2}$ respectively. This result is exact for elastic collisions (such as gravitational deflections); but Stewart et al. (1984) have shown that collision inelasticity prevents exact energy equipartition. However the correcting factor is of the order of $1+\epsilon_{\mathrm{r}}$, which does not modify our discussion. Suppose that a cold swarm of $M_{1}$ mass planetesimals collides with a swarm of $M_{2}$ mass fragments $\left(M_{1}>M_{2}\right)$ generated after some violent collisions. Random velocities of planetesimals and fragments are $V_{1}$ and $V_{2}$ respectively. Two different cases are possible:

1. If $V_{2} / V_{1}>\left(M_{1} / M_{2}\right)^{1 / 2}$, fragments are able to stir the cold disc of planetesimals. In consequence, the collisional diffusion may propagate but its extention may be less than observed in the present simulations. The following scenario may be possible: after the formation of a $15 M_{\oplus}$ Jupiter embryo, the part of the disc between 3 and 10 a.u. heats up due to violent collisions (see Fig. 4). Once all eccentric parent bodies have been disrupted, fragments maintain remaining planetesimals in a residual excited state;

2. If $V_{2} / V_{1}<\left(M_{1} / M_{2}\right)^{1 / 2}$, the random energy of the initially unperturbed planetesimal swarm is transferred to the swarm of fragments. In consequence the planetesimal disc cools down. The following scenario may be envisioned: like in the previous case, the region extending from 3.5 to 10 a.u. is first heated up via violent collisions. Later, the fragment population cools down the remaining planetesimal swarm. Consequently, two phases are present: a very thin and cold planetesimal disc embedded in a hot swarm of fragments.

These considerations emphasize the importance of the distribution of random velocities among different mass bodies for the dynamical evolution of the perturbed system.

Whereas equal-mass particles were considered in our simulations, it is expected that the size of planetesimals may increase with heliocentric distance (Lissauer et al. 1995; Pollack et al. 1996). For example Pollack et al. (1996) considers that planetesimal's radii are of $100 \mathrm{~km}$ at 5 a.u. when the Jupiter's core was still suffering runaway growth. Thus, heavy bodies deflected by the protoJupiter and that collide with kilometer-sized planetesimals in the inner Solar System may not suffer catastrophic fragmentation. They would suffer an erosion process instead, because of the $\sim 10^{3}$ to $10^{6}$ ratio in masses. Such collisions may produce numerous kilometer-sized fragments on highly eccentric orbits, thus increasing the efficiency of inward collisional diffusion. The study of these mechanisms requires a somewhat different numerical approach. This will be considered in the future.

\section{Potential application to the Solar System}

\subsection{Slowing down of runaway accretion processes}

Bearing in mind all previous considerations on the possibility for collisional diffusion to exist in the early Solar System, we now envision the potential consequences of this mechanism by doing the hypothesis that collisional diffusion actually occurs as observed in the simulations. The apparition of a proto giant-planet's core may slow down accretion processes of other runaway embryos in the perturbed region. As shown in Sect. 3, in the perturbed region particle random velocities rise from 100 to $1500 \mathrm{~m} / \mathrm{s}$, depending on their location and on the perturber's mass. Indeed, the minimal condition required to slow down runaway accretion is that the escape velocity of present runaway embryos be lower than rebound velocity among planetesimals. Assuming a typical rebound velocity of a few $100 \mathrm{~m} / \mathrm{s}$ in the perturbed region, the minimum mass of a runaway body must be $10^{23} \mathrm{~g}$ to $10^{24} \mathrm{~g}$ (or $100 \mathrm{~km}$ to $400 \mathrm{~km}$ radius) to maintain their accretion process even under the influence of collisional diffusion. This simple estimate neglects dynamical friction that may substantially lower this minimum mass. Thus, it is natural to ask the following question: what is the size of runaway embryos in the perturbed region when the collisional diffusion starts? It has been shown that collisional diffusion appears almost simultaneously with Jupiter's embryo. In consequence the previous question hides at least two other important questions, the answers to which are not currently known: 1. What is the typical growth timescale of runaway embryos in the perturbed region?

2 . When does a $15 M_{\oplus}$ proto-Jupiter appear?

Lissauer (1987) proposes a simple analytical expression of the runaway growth rate, valid at the beginning of runaway accretion:

$\frac{\mathrm{d} R}{\mathrm{~d} t}=\sqrt{\frac{3}{\pi}} \frac{\sigma \Omega(1+2 \theta)}{4 \rho}$

where $R$ is the embryo's radius, $\sigma$ the solid material surface density, $\rho$ the density of the embryo, $\Omega$ the Keplerian angular speed, and $\theta$ the gravitational correcting factor. Wetherill \& Cox (1985) show that at 3 a.u. (i.e. in the perturbed region below Jupiter's orbit) $\theta$ may be between 100 and 1000. From Eq. (6) an estimate of the growth timescale of a $200 \mathrm{~km}$ embryo $\left(T_{\mathrm{e}}\right)$ at 3 a.u. is $10^{5}$ to $10^{6}$ years, assuming a constant radius increase with 


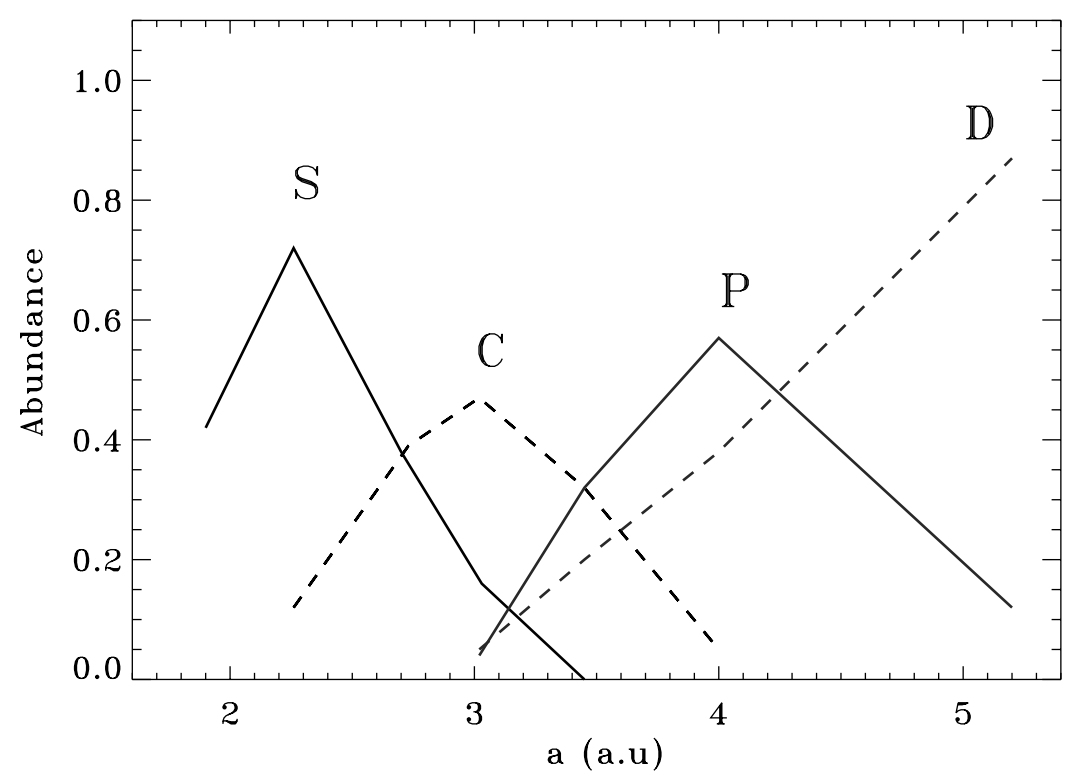

Fig. 14. Distribution of asteroid spectroscopic families from Gradie \& Tedesco (1982).

$\rho=3 \mathrm{~g} / \mathrm{cm}^{3}$ and $\sigma=1.2 \mathrm{~g} / \mathrm{cm}^{2}$ (Hayashi et al. 1981). For comparison, Wetherill (1992) assumes that $10^{26} \mathrm{~g} \mathrm{em}-$ bryos appear at 3 a.u. in $2 \times 10^{6}$ years, which also implies that $10^{23} \mathrm{~g}$ embryos appear in a few $10^{5}$ years, assuming a constant radius increase.

The answer to the second question is even more difficult. Concerning Jupiter's formation, one of the fundamental difference with the inner Solar System environment is the presence of ice beyond 4.5 a.u. (Lissauer 1987), which abruptly increases the surface density of solid material by a factor of 3 to 4 . A model of proto-Jupiter growth has been presented by Pollack et al. (1996) in which the Jupiter's core formation timescale (referred as $T_{\mathrm{j}}$ ) is about $5 \times 10^{5}$ years. More recently, some authors have suggested that Jupiter may have fully formed in a few $10^{6}$ years only, implying a very short timescale for the apparition of the core, of the order of $10^{5}$ years (D. Gautier, private communication).

In conclusion, it seems that $T_{\mathrm{e}}$ and $T_{\mathrm{j}}$ are of the same order. Due to numerous uncertainties that still exist about the formation processes of planetary embryos, and especially about initial conditions, it is not possible today to discard the possibility that a $10-15 M_{\oplus}$ Jupiter embryo may have appeared before the formation of 100 to $1000 \mathrm{~km}$ embryos at 3 a.u. from the Sun. In this case runaway growth of kilometer-sized planetesimals may have been slowed down or even completely stopped. In the opposing hypothesis of already present lunar sized bodies, accretion of the biggest bodies may not have been stopped but rather slowed down due to the simple effect of high impact velocities, unfavorable for gravitational sticking, switching from a "runaway growth" to a very slow "orderly growth" as described by Safronov (1969).

Regarding the outer disc beyond Jupiter, it is unlikely that collisional diffusion may have stopped accretion. Indeed, the mass of runaway embryos may increase with heliocentric distance (Lissauer et al. 1995). Thus, it is likely that a population of runaway bodies larger than $10^{24} \mathrm{~g}$ was already present between 5 and 10 a.u. at the time the $15 M_{\oplus}$ proto-Jupiter appeared. This population may have been little affected by the collisional diffusion triggered by the proto-Jupiter. However, an intense fragmentation cascade may have occurred as well as a substantial radial mixing of material.

\subsection{The Asteroid Belt}

\subsubsection{Main characteristics}

The origin of the Asteroid Belt is still a puzzle. Its main characteristics that have to be explained by an adequate model, are the following:

1. The absence of any large body (Ceres's radius is only $\sim 450 \mathrm{~km}$ );

2. A strong dynamical excitation. Eccentricities and inclinations are 0.15 on average, with a dispersion of 0.1 ;

3. A strong radial mixing. Spectroscopic studies of Asteroid families show that they are mixed over a distance of a few 0.1 a.u. typically as shown by Gradie \& Tedesco (1982) (see Fig. 14). This contradicts the simple model of the spatial zoning of spectroscopic types due to the initial temperature gradient in the protoplanetary disc;

4. A large mass depletion. By comparison to the model of minimum mass nebula, about $99.9 \%$ of solid material initial mass may have disappeared (Weidenschilling 1977).

\subsubsection{Possible role of collisional diffusion}

All models that have been proposed so far require one way or another the presence of Jupiter with its current 


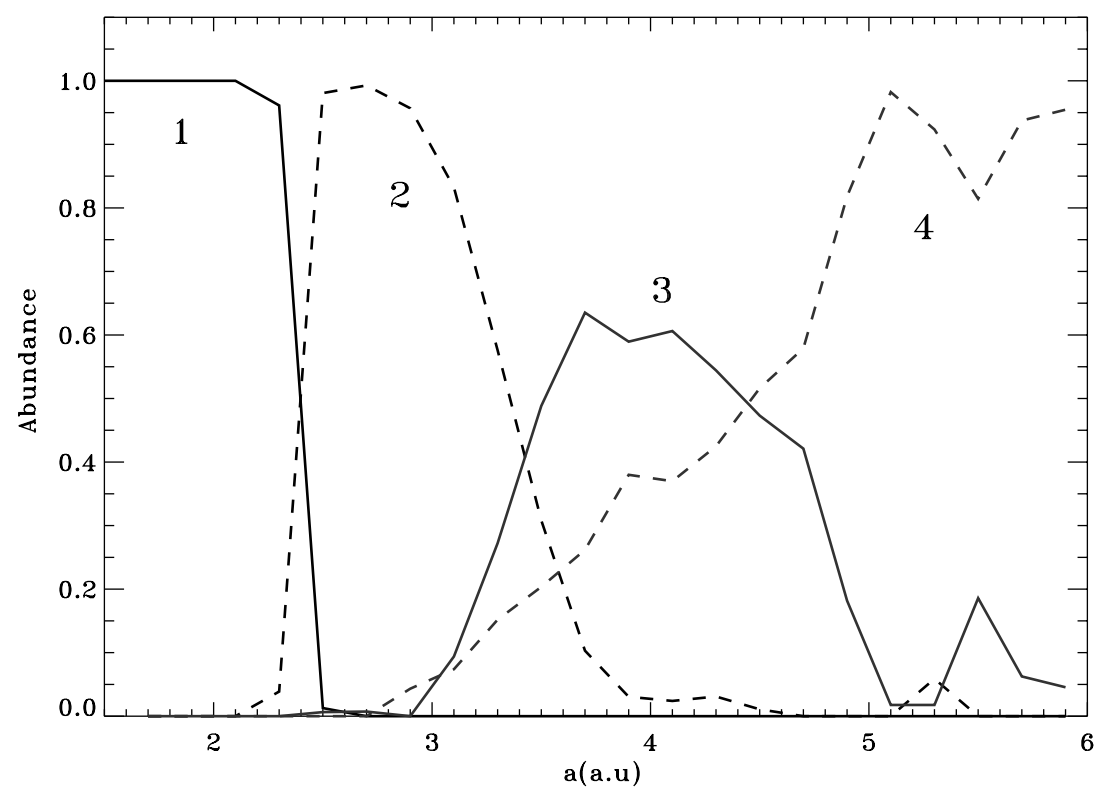

Fig. 15. Local abundances of types $1,2,3$ and 4 families at the end of the collisional diffusion triggered by a $15 M_{\oplus}$ body.

mass: via the coupling with Saturn and the dissipation of the solar nebula (Lecar \& Franklin 1997; Nagasawa et al. 2000), via the coupling with large scattered planetesimals (Wetherill 1989; Petit et al. 1999) and via the coupling with initially present protoplanets (Wetherill 1992; Petit et al. 2000).

It is suggested hereafter that some characteristics of the Asteroid Belt may be explained by the early presence of a mere $15 M_{\oplus}$ Jupiter embryo. Possible consequences of a collisional diffusion in the early Asteroid Belt triggered by a $15 M_{\oplus}$ Jupiter embryo are reviewed here.

1. As shown in Sect. 6.1, if collisional diffusion was active during the formation of the Asteroid Belt, it may have slowed-down planetary accretion within it. This may be a partial answer to the problem of the absence of a fully-grown planet in this region. The biggest runaway body might be of a few hundred kilometers, which is qualitatively consistent with what is observed today;

2. Figure 6 shows that in the region of the Asteroid Belt, the collisional diffusion triggered by a $15 M_{\oplus}$ perturber may increase eccentricities and inclinations up to 0.05 at most, which is at least two or three times lower than what is currently observed. A strong gradient of eccentricities and inclinations also appears in simulations, but is not observed in the actual Asteroid Belt. These arguments make unlikely that the current excited dynamical state of the Asteroid Belt is the result of an early collisional diffusion. However, this conclusion does not imply that collisional diffusion never happened, as the present study only deals with the first $10^{6}$ years of the Asteroid Belt. The latter may have been deeply modified over 4.5 billions years of evolution, under the influence of giant planets (Brunini 1988; Ries 1996);

3. Numerical simulation gives interesting results concerning the radial mixing of spectroscopic asteroid families.
The particles were flagged according to their initial semi-major axes into four distinct populations. The type 1 family extends initially below 2.5 a.u., the type 2 family extends from 2.5 to 3.5 a.u., the type 3 family extends from 3.5 to 4.5 a.u. and the type 4 family extends beyond 4.5 a.u. The resulting radial distribution after the propagation of collisional diffusion is presented in Fig. 15 and must be compared to Fig. 14. Families of types 1 and 2 slightly overlap because they are far away from the perturber, and thus are little affected by the collisional diffusion. The type 3 family is qualitatively consistent with the actual $\mathrm{P}$ spectroscopic family: it spreads widely in both inward and outward directions. However, the most interesting result relates to the family of type 4 , the abundance profile of which is very similar to the actual D spectroscopic family: it is predominant at 5 a.u. and decreases linearly down to 0 at about 3 a.u.;

4. Regarding the large mass depletion, the collisional diffusion may not by itself constitute an explanation: within the assumptions of our model (i.e. no fragmentation, equal-mass bodies, no gravitational deflection) no significant mass depletion is observed. However, the coupling between fragmentation and gas drag, which has not been considered here, may provide a mechanism that depletes the system's mass, when associated with collisional diffusion. Indeed gas drag eliminates sub-kilometer fragments on short time-scales. This will be part of some future works.

In conclusion, it does not seem possible that collisional diffusion explains by itself every characteristic of the Asteroid Belt. However, some of them may be a signature of an early collisional diffusion triggered by a $15 M_{\oplus}$ proto-Jupiter, in particular the observed radial mixing of asteroid spectroscopic families. This may suggest that the zoning of asteroid spectroscopic asteroid families may 
be anterior to the apparition of Jupiter with its present mass. Thus, it might be possible that collisional diffusion occurred in the early Asteroid Belt, shaping the structure of spectroscopic asteroid families, and that another subsequent mechanism depleted the system's mass and increased eccentricities and inclinations, as suggested by Lecar \& Franklin (1997); Wetherill (1989) or Wetherill (1992).

\section{Conclusion}

Using a simple model of a disc of colliding particles perturbed by a giant-planet embryo at 5.2 a.u., the coupling of inelastic rebounds among particles with the embryo's gravitational perturbations has been investigated. The perturber's mass is in the range from $0.5 M_{\oplus}$ to $300 M_{\oplus}$, paying a deeper attention to the case of a $10-15 M_{\oplus}$ body, compatible with the "solid-core" model of Jupiter's formation. Initially the disc is cold: particle random velocities are of $10 \mathrm{~m} / \mathrm{s}$, comparable to kilometer-sized planetesimal escape velocity. Once inelastic collisions and protoplanet's gravitational perturbations are introduced, the system shows major modifications. The main effects are the following:

- A heat transfer, or "collisional diffusion" is triggered in the vicinity of the perturber's orbit and propagates away from it. The heated region extends over 2 to 3 a.u. inwards and at least over 4 a.u. outwards;

- The propagation timescale is typically a few $10^{5}$ years inwards and about 5 times longer outwards for a comparable particle surface density. This timescale decreases with the perturber's mass: it drops to a few $10^{4}$ years for the inward diffusion triggered by a $300 M_{\oplus}$ perturber;

- In the perturbed region, average random velocities range from 100 to $1000 \mathrm{~m} / \mathrm{s}$, decreasing away from the perturber;

- The radial mixing length-scale ranges from a few 0.1 to 1 a.u.;

- The extent of the perturbed region increases with the perturber's mass and is quantified in Fig. 13 and in Table 1.

Bearing in mind the limited scope of this model (see Sect. 2), potential consequences of collisions on planetary accretion can be drawn: collisional diffusion may slow down runaway accretion processes in the region of the Asteroid Belt as soon as a $15 M_{\oplus}$ Jupiter embryo is formed; radial mixing of particles is qualitatively consistent with the actual mixing of the asteroid spectroscopic families. These points suggest that collisional diffusion plays a role in the early evolution of the Asteroid Belt. However, this statement may be revised in light of two opposite effects taking place in the actual early Solar System. Fragmentation might act as an "energy well" preventing the propagation of collisional diffusion. Conversely, an increased planetesimal size in the proximity of Jupiter (larger than in the inner Solar System, as used by Pollack et al. 1996), should increase the efficiency of diffusion to heat-up the inner Solar System. Unfortunately, the true initial size distribution of planetesimals, as a function of heliocentric distance, is highly unknown. In any case, collisional diffusion has proved to be a potentially effective mechanism that must be included in studies of planetary formation. The open issues are the following:

- What is the long-term evolution of the collisional diffusion?

- What is the fate of coorbital objects? Are they stable against collisional diffusion?

- What is the impact of gas drag?

- What is the impact of fragmentation and mass distribution?

Some of these questions will be investigated in a forthcoming paper.

Acknowledgements. The authors are pleased to thank A. Morbidelli, J.-M. Petit and D. Richardson for valuable comments.

\section{References}

Barge, P., \& Pellat, R. 1990, Icarus, 85, 481

Beaugé, C., \& Aarseth, S. J. 1990, MNRAS, 245, 30

Brunini, A. 1988, MNRAS, 293, 405

Benz, W., \& Asphaug, E. 1999, Icarus, 142, 5

Brahic, A. 1976, Jour. Comp. Phys., 22, 171

Giblin, I., Martelli, G., Smith, P. N., \& Di Martino, M. 1994, Planet. Space Sci., 42, 1027

Gradie, J., \& Tedesco, E. 1982, Science, 216, 1405

Greenberg, J. M., Wacker, J. F., Hartmann, W. L., \& Chapman, C. R. 1978, Icarus, 35, 1

Hanninen, J., \& Salo, H. 1992, Icarus, 97, 228

Hanninen, J., \& Salo, H. 1994, Icarus, 108, 325

Hayashi, C., Nakazawa, K., \& Adachi, I. 1977, Publ. Astron. Soc. Jpn., 29, 163

Hayashi, C. 1981, Prog. Theor. Phys. Suppl., 70, 35

Hertzsch, J.-M., Scholl, H., Spahn, F., \& Katzorke, I. 1997, A\&A, 320, 319

Ida, S., \& Makino, J. 1993, Icarus, 106, 210

Kokubo, E., \& Ida, S. 2000, Icarus, 143, 15

Kortenkamp, S. J., \& Wetherill, G. W. 2000, Icarus, 143, 60

Lecar, M., \& Franklin, F. 1997, Icarus, 129, 134

Lissauer, J. J. 1987, Icarus, 69, 249

Lissauer, J. J., Pollack, J. B., Wetherill, G. W., \& Stevenson, D. J. 1995, In Neptune and Triton ed. D. Cruikshank, (University of Arizona Press, Tucson), 37

Martelli, G., Ryan, E. V., Nakamura, A. M., \& Giblin, I. 1994, Planet. Space Sci., 42, 1013

Marzari, F., \& Scholl, H. 1997, PSS, 45, 337

Marzari, F., \& Scholl, H. 2000, ApJ, 543, 328

Murison, A. 1989, AJ, 97, 1496

Nagasawa, M., Tanaka, H., \& Ida, S. 2000, AJ, 119, 1480

Nakamura, A., \& Fujiwara, A. 1991. Icarus, 92, 132

Petit, J.-M., \& Farinella, P. 1993, CeMDA, 57, 1

Petit, J.-M., Morbidelli, A., \& Valsecchi, G. B. 1999, Icarus, 141,367 
Petit, J.-M., Morbidelli, A., \& Chambers, J. 2000, submitted to Icarus

Pollack, J. B., Hubickyj, O., Bodenheimer, P., et al. 1996, Icarus, 124,62

Press, W. H., Teukolsky, S. A., Vetterling, W. T., \& Flannery, B. P. 1992, Numerical Recipes in Fortran (Cambridge University Press)

Ries, J. G. 1996, Icarus, 121, 202

Richardson, D. C., Quinn, T., Stadel, J., \& Lake, G. 2000, Icarus, 143, 45

Safronov, V. S. 1969, in Evolution of the protoplanetary cloud and formation of the Earth and planets (Moscou: Nauka Press)

Spaute, D., Weidenschilling, S. J., Davis, D. R., \& Marzari, F. 1991, Icarus, 92, 147

Stern, S. A., \& Weissman, P. R. 2001, Nature, 409, 589
Stewart, G. R., Lin, D. N. C., \& Bodenheimer, P. 1984, in Planetary rings, ed. Greenberg \& Brahic (Tucson: Univ. of Arizona Press), 447

Tanaka, H., \& Ida, S. 1996, Icarus, 120, 371

Thébault, P., \& Brahic, A. 1999, Planet. Space Sci., 47, 233

Trulsen, J. 1971, AP\&SS, 12, 239

Weidenschilling, S. J. 1977, Astrophys. Space Sci., 180, 57

Wetherill, G. W., \& Cox, L. P. 1985, Icarus, 60, 40

Wetherill, G. W., \& Stewart, G. R. 1989, Icarus, 77, 330

Wetherill, G. W. 1989, in Asteroids II, ed. R. P. Binzel, T. Gehrels, \& M. S. Matthews (Tucson: Univ. of Arizona Press), 661

Wetherill, G. W. 1992, Icarus, 100, 307

Wisdom, J. 1980, A\&A, 85, 1122

Wisdom, J., \& Holman, M. 1991, AJ, 102, 1528 